DOI: $10.19195 / 0524-4544.327 .24$

\author{
WOLFGANG SCHRÖDER \\ ORCID: 0000-0003-4006-3367
}

Brandenburgische Technische Universität Cottbus-Senftenberg wolfgang.schroeder@b-tu.de

\title{
Die allmähliche Preisgabe des Nationalstaates: Von „Kongresspolen“" zum „Weichselgouvernement" — gibt es Parallelen im Hinblick auf EU-Rechtssetzungen und deren Vollzug? \\ Betrachtungen zu staatlicher Souveränität und Identität im Verhältnis zu supranationalem Recht am Beispiel Polen
}

Zusammenfassung: Der Artikel betrachtet angesichts der historischen Erfahrungen Polens mit der über 100jährigen Herrschaft ausländischer Souveräne, insbesondere der russischen von 1815 bis 1918, die heutigen Vorbehalte Polens gegen die Gefahr einer ausufernden Verlagerung nationaler Souveränitätsrechte auf Institutionen der Europäischen Union. Teilaspekte dieser Verlagerung tangieren nach Auffassung des Unterzeichners bestimmte Grundsätze des europäischen Primärrechts.

Schlüsselwörter: Souveränität, Nationalstaat, Kultur, Europäische Union, Fremdherrschaft.

\section{A. Vorgeschichte}

\section{Westeuropas Rettung und Undank}

Im Jahre 1683 hatten die Hussaria (Flügelhusaren) der Rzeczpospolita Korony Polskiej i Wielkiego Księstwa Litewskiego (Republik der polnischen Krone und des 
Großfürstentums Litauen) unter ihrem König Jan III Sobieski entscheidend zum Sieg des Defensivbündnisses zwischen dem Heiligen Römischen Reich, eben Polen-Litauen sowie der Republik Venedig über die osmanischen Belagerer Wiens bei der Schlacht am Kahlenberg beigetragen ${ }^{1}$. Durch diesen Sieg vor Wien, dem „Tor zu Westeuropa“ oder dem „Goldenen Apfel der Deutschen“2, wie die Osmanen es nannten, wurde das christliche Abendland zum zweiten Mal - nach 1529 vor der Eroberung durch das aggressiv-expansionistische Osmanische Reich und damit vor der Zwangsunterwerfung unter den Islam, sei es durch Konvertitentum oder Dhimmitude ${ }^{3}$, bewahrt. Bis die weiterhin besetzten Teile Europas von der osmanischen Unterjochung endgültig befreit wurden, sollten indes noch fast 200 Jahre vergehen ${ }^{4}$.

In der Folge der gescheiterten zweiten türkischen Belagerung Wiens wurde der polnischen Nation ihr erfolgreicher Einsatz für Europa, insbesondere für das Heilige Römische Reich, allerdings schlecht vergolten, denn schon 1772 geschah die Erste Teilung Polens, 1793 die Zweite und 1795 die Dritte. Von der Ersten und der Dritten Teilung ,profitierten“ territorial und bevölkerungsmäßig die Teilungsmächte, das Königreich Preußen, das österreichische und das russische Kaiserreich, von der Zweiten „nur“ Preußen und Russland 5 .

\section{Polens Hoffnung}

Während der napoleonischen Ära wurde 1807 aufgrund der Art. 15 ff. des Friedensvertrages von Tilsit ${ }^{6}$ eine polnische Staatlichkeit in Gestalt des Księstwo Warszawskie (Herzogtum Warschau) errichtet und zum Herzog der sächsische König Friedrich August I. ernannt. So verband die von Napoleon am 22. Juli 1807 zu Dresden diktierte Verfassung ${ }^{7}$ für das Herzogtum in ihrem Artikel 5 weiterhin - hierin anknüpfend an die polnische Verfassung von $1791^{8}$, nach der Friedrich August in jenem Jahr schon zum König von Polen ernannt worden war - die Warschauer Herzogswürde erblich mit dem sächsischen Königshaus. Dieses Herzogtum wird oft als französisch-napoleonischer Satelliten- und polni-

1 https://www.welt.de/geschichte/article149358500/Polens-Panzerreiter-retteten-Wien-vorden-Tuerken.html, zuletzt abgerufen am 14.12.2018.

2 Nach Konstantinopel, dem ersten ,goldenen Apfel“" und Belgrad, dem ,goldenen Apfel der Ungarn“, https://www.alaturka.info/de/geschichte/mittelalter/1038-der-goldene-apfel-wien, zuletzt abgerufen am 14.12.2018.

3 https://en.wikipedia.org/wiki/Dhimmitude, zuletzt abgerufen am 14.12.2018.

4 Nach dem Berliner Kongress 1878, https://www.bundestag.de/blob/413460/941d900baae61 2b71736779e68900a3d/wd-1-145-05-pdf-data.pdf, s. 21 ff., zuletzt abgerufen am 14.12.2018.

5 https://de.wikipedia.org/wiki/Teilungen_Polens m.w.N., zuletzt abgerufen am 14.12.2018.

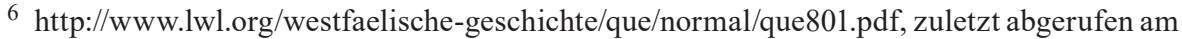
14.12.201, http://www.preussenweb.de/friede.htm, zuletzt abgerufen am 14.12.2018.

7 http://www.verfassungen.eu/pl/verf07-i.htm, zuletzt abgerufen am 14.12.2018.

8 http://www.verfassungen.eu/pl/verf91-i.htm, zuletzt abgerufen am 14.12.2018. 
scher Rumpfstaat bezeichnet und bestand im Wesentlichen zunächst aus dem Teil Polens, der von Preußen bei der Zweiten und Dritten Teilung Polens annektiert worden war; er wurde 1809 um das von Österreich in der Dritten Teilung annektierte polnische Gebiet Westgalizien erweitert. Dass Polen bis heute dieses Herzogtum - und damit auch Napoleon - als ein Positivum empfand und empfindet, weil es und seine militärischen Repräsentanten die Hoffnung auf einen polnischen Nationalstaat verkörperte, kann etwa diesem Textteil der aktuellen polnischen Nationalhymne entnommen werden: „Marsz, marsz, Dąbrowski, z ziemi włoskiej do Polski. Za twoim przewodem złaczym się z narodem. Przejdziem Wisłe, przejdziem Wartę, będziem Polakami. Dat nam przykład Bonaparte, jak zwyciężać mamy." (,Marsch, marsch, Dąbrowski, von der italienischen Erde nach Polen. Unter deiner Führung vereinen wir uns mit der Nation. Wir werden Weichsel und Warthe durchschreiten, wir werden Polen sein, Bonaparte gab uns ein Beispiel, wie wir zu siegen haben." $)^{9}$. Schon 1796 hatte Jan Henryk Dąbrowski in Frankreich militärische Verbände von polnischen Freiwilligen organisiert. Diese hatten sich in den Dienst der Französischen Republik gestellt und ihr Ziel war es gewesen, mit Hilfe Frankreichs ehemals polnische Gebiete zu befreien ${ }^{10}$.

\section{Wiener Kongress}

Das Herzogtum Warschau endete 1815. Vorausgegangen waren die Niederlage Napoleons in der Völkerschlacht bei Leipzig 1813, die Proklamation Ludwig XVIII., des Bruders von Ludwig XVI., zum König der Franzosen durch den französischen Senat am 7. April $1814^{11}$, die im Vertrag von Fontainebleau mit Österreich, Russland und Preußen vereinbarte Abdankung Napoleons am 11. April $1814^{12}$, der „Erste Pariser Frieden“ vom 30./31. Mai 1814 zwischen dem Frankreich Ludwigs XVIII. einerseits und den alliierten Mächten Österreich, Großbritannien, Preußen, Russland, Spanien, Portugal sowie Schweden und Norwegen andererseits $^{13}$; nach dessen Art. 32 wurde dem Wiener Kongress die endgültige Erledigung der vorläufigen (territorialen) Bestimmungen übertragen.

Auf diesem europäischen Gipfel, der von September 1814 bis Juni 1815 dauerte, waren die Signatarstaaten des Ersten Pariser Friedens, des weiteren 33 deutsche Staaten, 12 nichtdeutsche souveräne (trotz noch immer großer besetzter Gebiete

9 https://de.wikipedia.org/wiki/Mazurek_D\%C4\%85browskiego\#\%C3\%9Cbersetzung_ins_ Deutsche, zuletzt abgerufen am 14.12.2018.

10 https://www.britannica.com/place/Poland/Partitioned-Poland\#ref396907, zuletzt abgerufen am 14.12.2018.

11 https://de.wikipedia.org/wiki/Ludwig_XVIII.\#Wahl_zum_\%E2\%80\%9EK\%C3\%B6nig _ der_Franzosen $\%$ E2\%80\%9C, zuletzt abgerufen am 14.12.2018.

12 https://fr.wikisource.org/wiki/Trait\%C3\%A9_de_Fontainebleau_(1814), zuletzt abgerufen am 14.12.2018.

13 http://www.staatsvertraege.de/Frieden1814-15/1pfv1814-i.htm, zuletzt abgerufen am 14.12.2018. 
Südosteuropas durch das osmanische Reich ohne dieses) oder früher souveräne Staaten vertreten, auch nahmen noch insgesamt 95 Delegationen des (mediatisierten) Reichsadels und anderer Gruppen mit partikularen Interessen teil ${ }^{14}$. Polen war indes offiziell in keiner Delegation vertreten, und auch dem sächsischen König und (immer noch) Herzog von Warschau, Friedrich August, war - anders als etwa den Vertretern Frankreichs - die Teilnahme untersagt. Es hieß, er sollte gleichsam stellvertretend für die Bündnispartner Napoleons abgestraft werden. Hinter beiden Maßnahmen stand freilich nichts anderes als die Absicht Preußens und Russlands, ihre schon am 28. Februar 1813 in dem Vertrag von Kalisch ${ }^{15}$ vereinbarten Annexionspläne durchzusetzen. Danach wollten sich Preußen mit ganz Sachsen und Russland mit dem Gebiet des Herzogtums Warschau „belohnen“. Dass es dann doch nicht zur völligen Aufgabe Sachsens kam, lag an der Furcht Österreichs, Frankreichs und Großbritanniens vor einem übermäßigen Erstarken Preußens. Nachdem der Kongress an der sächsischen Frage zu zerbrechen drohte und der Konflikt eskalierte, kam man schließlich auf Vermittlung des Zaren überein, Sachsen zu teilen; das Schicksal Polens geriet damit als das eines „Verliererstaates“ offenbar in den Hintergrund. So konnte der Plan Alexanders I., auf dem Gebiet des Herzogtums Warschau ein polnisches Königreich unter russischer Herrschaft $\mathrm{zu}$ errichten, weitgehend umgesetzt werden. Dass zu diesem Zeitpunkt Polen immerhin den Status eines Königreiches erhalten sollte, war wohl nicht zuletzt dem polnischen Fürsten Adam Jerzy Czartoryski zu verdanken, der — von 1804 bis 1806 russischer Außenminister - den Zaren zum Wiener Kongress begleitete und von dem es heißt, er habe sich als Freund Alexanders I. erfolgreich für eine Verfassung des Königreichs Polen eingesetzt und diese auch mitformuliert ${ }^{16}$.

\section{B. „Kongresspolen“}

\section{Wiener Schlussakte und Verfassung des Königreichs Polen}

Die Verhandlungen auf dem Wiener Kongress mündeten in die Schlussakte vom 9. Juni $1815^{17}$, die von den bevollmächtigten Vertretern der Signatarstaaten

$14 \mathrm{https} / / /$ de.wikipedia.org/wiki/Wiener_Kongress\#Delegationen_und_bedeutende_Teilnehmer, zuletzt abgerufen am 14.12.2018.

15 https://de.wikipedia.org/wiki/Vertrag_von_Kalisch_(1813), zuletzt abgerufen am 14.12.2018.

$16 \mathrm{https}$ :/de.wikipedia.org/wiki/Wiener_Kongress\#Die_Verhandlungen, zuletzt abgerufen am 14.12.2018.

17 https://opacplus.bsb-muenchen.de/Vta2/bsb10556621/bsb:4262295?page=5, zuletzt abgerufen am 14.12.2018; http://www.staatsvertraege.de/Frieden1814-15/wka1815-i.htm, zuletzt abgerufen am 14.12.2018. 
des „Ersten Pariser Friedens“ (außer Spanien) unterzeichnet wurde. Sie bestand aus 121 Artikeln sowie 17 bi-, tri- und multilateralen Staatsverträgen zwischen Teilnehmerstaaten des Kongresses, die nach Art. 118 der Schlussakte als Bestandteil derselben galten. In Bezug auf Polen waren die Artikel 1 bis 14 der Schlussakte, der Vertrag zwischen Russland und Preußen vom 21. April/3. Mai 1815 (Anlage 2 zur Schlussakte mit 43 Artikeln) und der Zusatzvertrag gleichen Datums zwischen Österreich, Preußen und Russland in Bezug auf das Gebiet Krakau (Anlage 3 zur Schlussakte) maßgebend. Jeweils erster und bestimmender Artikel der Schlussakte sowie des russisch-preußischen Vertrages war die Bestimmung, dass das Gebiet des Herzogtums Warschau mit Russland „vereint“" (,réuni“) werde, mit Ausnahme der Gebiete, die Preußen („Großherzogtum Posen“) und Österreich (Tarnopol und umliegende Kreise) zugeordnet wurden oder eine freie Stadt bildeten (Krakau und Umgebung). Weiter bestimmte Art. 1 (Abs. 1 Sätze 2 ff. und Abs. 2) der Schlussakte, der nahezu wortgleich mit Art. 3 des russisch-preußischen Vertrages übereinstimmte bzw. von diesem übernommen wurde:

Unwiderruflich wird es [das verbleibende Gebiet des Herzogtums Warschau - W. Sch.] durch seine Constitution mit demselben [Russland - W. Sch.] verbunden, um auf ewige Zeiten im Besitze Sr. Majestät des Kaisers aller Reussen, seiner Erben und Nachfolger zu verbleiben. Se. kaiserl. Majestät behalten sich vor, diesem Staate, welcher sich einer besonderen Administration zu erfreuen hat, diejenige innere Ausdehnung zu geben, welche sie für ihn passend halten werden. Sie nehmen zu den mit ihren übrigen Besitzungen verbundenen Titeln auch den eines Czars und Königs von

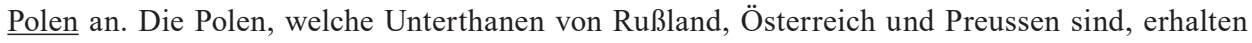
Ständeversammlungen und nationale Einrichtungen, der politischen Existenz gemäß, welche die Regierungen, denen sie angehören, für nützlich und zweckmäßig halten werden.

Die in beiden Dokumenten angesprochene bzw. vorgegebene Verfassung wurde vom Zaren am 27. November $1815^{18}$ verordnet und manifestierte damit die Vierte Teilung Polens, weil die Souveränität Polens wieder auf nichtpolnische Souveräne, nämlich die jeweiligen Herrscher Russlands, Preußens und Österreichs aufgeteilt worden war, die sich im Übrigen auch nach Art. 6 der Schlussakte und dem trilateralen Vertrag über das Gebiet Krakau als dessen „Schutzmächte“ installiert hatten ${ }^{19}$.

Nach dieser Verfassung sollte das ,neue“ Königreich Polen eine relativ starke Autonomie besitzen, z.B. hatte der Sejm (= Reichstag oder Landtag) die zivil-, straf- und verwaltungsrechtliche Gesetzgebungs- sowie die Haushaltskompetenz (Art. 31, 90 und 91), wobei der noch unter Napoleon im Herzogtum Warschau eingeführte Code Civil sogar bis 1915 Gültigkeit behielt, es wurde eine eigenständige Armee ( $\S 153$ ff.) und Währung ( $(91)$ beibehalten. Die Amtssprache war ausnahmslos polnisch (§ 28) und die öffentlichen Ämter mit Ausnahme das des Königs als Souverän und Staatsoberhaupt und seines Statthalters (falls kein „kaiserlicher Prinz von Russland“" ernannt wird, § 6) waren dem polnischen Adel bzw. anderen

18 http://www.verfassungen.eu/pl/verf15-i.htm, zuletzt abgerufen am 14.12.2018.

19 Siehe Fn. 17. 
polnischen Staatsbürgern vorbehalten (§ 29). Hinsichtlich Weiterentwicklung (und Änderung) dieser Verfassung verwies $§ 161$ auf künftige „organische Statuten“20. In diese Zeit fiel auch die Gründung der Universität Warschau im Jahre 1816. All diese Regelungen und Maßnahmen ließen das Königreich Polen jedenfalls als freiesten Teil der vom russischen Zaren beherrschten Gebiete erscheinen.

\section{Verfassungseinschränkungen}

Diese Verfassungsfreiheiten und damit auch die Regelungen der Schlussakte des Wiener Kongresses wurden aber sukzessive außer Kraft gesetzt. Schon 1819 erfolgte entgegen $\S 16$ der Verfassung die Abschaffung der Pressefreiheit und die Einführung einer Zensur. Das 50000 Mann starke polnische Heer wurde dem Großfürsten Konstantin (Bruder und Statthalter des Zaren und faktisch Vizekönig von Polen) unterstellt und verschiedene Offiziersverbindungen sowie nationale Vereinigungen polnischer Bürger wurden in der Zeit 1821 und 1822 ausgehoben und verboten ${ }^{21}$. Mit zaristisch-königlichem Dekret vom 1./13. Februar 1825 wurde, entgegen $§ 95$ der Verfassung, die Öffentlichkeit der Verhandlungen des Sejm durch Übertragung vieler Zuständigkeiten auf einen geheimen Ausschuss faktisch aufgehoben ${ }^{22}$ :

Zusatzartikel zur Verfassungsurkunde. Die Eröffnungs- und Schlußsitzungen des Reichstags, so wie auch diejenigen, wo die königliche Sanction der Gesetzentwürfe promulgirt [öffentlich bekanntgemacht - W. Sch.] wird, sollen fernerhin, wie bisher, öffentlich seyn, und dabei die gewöhnlichen Feierlichkeiten stattfinden. Für die Wahl der Commissionen, sowie für sonstige Berathungen und Discussionen aller Art, sollen sich die Kammern immer in einen geheimen Ausschuß bilden. Dieser Artikel wird für einen integrirenden und untrennbaren Theil der Verfassungsurkunde erklärt.

Hierzu wäre eigentlich ein organisches Statut unter Zustimmung der Kammern des Sejm erforderlich gewesen, § 163 Satz 2 der Verfassung, was aber nicht erfolgte.

\section{Zunehmende Spannungen ${ }^{23}$}

Diese zunehmende Aushöhlung der zwischen den europäischen Großmächten vereinbarten und in der polnischen Verfassung von 1815 konkretisierten relati-

20 Siehe Fn. 18 und A.N. Makarov, Die russisch-polnischen Rechtsbeziehungen seit 1815 unter spezieller Berücksichtigung der Staatsangehörigkeitsfragen, ZaöRV 1929, S. 330 ff, http://www. zaoerv.de/01_1929/1_1929_1_a_330_367.pdf, zuletzt abgerufen am 14.12.2018.

21 Siehe Fn. 18 (Zusatzkommentar a. E.).

$22 \mathrm{http} / / / w w w . v e r f a s s u n g e n . e u / p l / v e r f 15-i . h t m$, zuletzt abgerufen am 14.12.2018.

23 Die folgenden Darstellungen zu B. 3 und 4. sowie zu C. beruhen im Wesentlichen auf Malte Rolf, Imperiale Herrschaft im Weichselland, Berlin-München-Boston 2015, insbesondere auf Teil II, https://books.google.de/books?id=hS11BgAAQBAJ\&pg=PA436\&lpg=PA436\&dq=we 
ven Autonomie des Königreiches Polen unter dem russischen Zarentum führte zu wachsendem Unmut in der polnischen Bevölkerung. Mitentscheidend war dabei eine noch repressivere Politik nach dem Petersburger Dekabristenaufstand nicht nur in Russland, sondern gerade auch in Polen, die der neue Zar Nikolaus I. betrieb, nachdem er seinem am 1. Dezember 1825 verstorbenen, kinderlosen Bruder Alexander I. unerwartet auf dem Thron gefolgt war, da der zweitälteste der Zarenbrüder, Konstantin, aufgrund einer „nicht standesgemäßen“ Ehe auf die Thronfolge verzichtet hatte. Die Irritationen, die wegen dieses genealogischen Wechsels im russischen Adels- und Offizierskorps entstanden waren, sollten einen Teil der Ursache für den brutal unterdrückten Dekabristenaufstand gebildet haben.

Dadurch kumulierte auch das gegenseitige Misstrauen auf polnischer und auf russisch-imperialer Seite. Im polnischen Adel verdichtete sich der Gegensatz zwischen der „roten“, also der mittleren und niederen szlachta und deren „weißer“, hochadliger Fraktion. Nur letztere war bereit, mit den Teilungsmächten zu kooperieren, während die „rote“ Fraktion weitaus überwiegend die Loslösung Polens von Russland anstrebte. Die weitere Verschärfung der Zensur nach 1825, die sich häufende Nichtachtung bzw. Verletzung der Verfassung von 1815 durch russische Amtsträger, die Zurückdrängung des Polnischen als Unterrichtssprache im - vormals polnisch-litauischen, nach 1792 russisch annektierten - Bezirk Vilnius, die fast vierjährige Dauer, bis sich Nikolaus I. nach Warschau begab, um sich dort zum König von Polen ausrufen zu lassen, die Schließung des vierten (und letzten) Sejms im Mai 1830 nach wenigen Wochen aufgrund „oppositioneller Haltung“ und letztlich eine Verhaftungswelle gegen polnisch-studentische „Aufrührer", führte zum sog. Novemberaufstand der Polen. Dieser begann am 29. November 1830, führte zur vorübergehenden Absetzung des Zaren als König von Polen und endete erst mit der Einnahme der Stadt Warschau durch russische Truppen am 18. September 1831.

\section{Organisches Statut}

Danach wandelte sich der bisherige, zumindest noch verfassungsmäßig mit relativ (s.o.) großer Autonomie ausgestattete Status Polens als staatsrechtlich selbständiges Königreich mit dem in Personalunion als König von Polen herrschenden russischen Zaren grundlegend. Mit Dekret des Zaren vom 1./13. Februar 1832 erfolgte die Ersetzung der Verfassung des Königreiches durch ein Organisches Statut $^{24}$, wodurch insbesondere die Legislative, der Sejm abgeschafft bzw. durch einen Staatsrat mit ernannten Mitgliedern ersetzt (Art. 28 ff. des Statuts) und die

ichselland\&source=bl\&ots=OY9-RsIfLA\&sig=a2XOsNSWlcV7UoUsYGnmRrZXPhY\&hl=de\& $\mathrm{sa}=\mathrm{X} \& \mathrm{ved}=0 \mathrm{ahUKEwjNo5KVwrnbAhUEZ1AKHTdTDZcQ6AEIbzAM} \# \mathrm{v}=$ onepage $\& \mathrm{q}=$ weichs elland\&f=false, zuletzt abgerufen am 14.12.2018.

$24 \mathrm{http} / / /$ www.verfassungen.eu/pl/verf32-i.htm, zuletzt abgerufen am 14.12.2018. 
eigenständige polnische Armee aufgelöst wurde (Art. 20 des Statuts). Die Krönung eines künftigen Königs von Polen sollte in Moskau gleichzeitig mit der Proklamation zum Zaren erfolgen, wobei Polen eine Delegation entsenden durfte (Art. 3 des Statuts). Insoweit wurde wiederum $\S 163$ Satz 2 der Verfassung des Königreiches verletzt, da der Sejm, der seine Zustimmung zum Statut hätte beschließen müssen, ab Mai 1830 eben gar nicht mehr einberufen worden war.

Wegen des nach 1830 in Permanenz verhängten Ausnahmezustandes und der Ausrufung des Kriegsrechts im Jahre 1833 durch Nikolaus I. blieb sogar das in Sachen polnische Autonomie gegenüber der Verfassung schon ausgehöhlte Organische Statut bis 1855 weitgehend rechtliche Makulatur ${ }^{25}$. Zudem hatte der Zar 1831 den siegreichen Befehlshaber der russischen Truppen gegen den Novemberaufstand, Feldmarschall Iwan Paskewitsch, zum Fürsten von Warschau erhoben und zu seinem Statthalter in Polen ernannt. Die ersten Amtshandlungen Paskewitschs waren auf die Bestrafung der Aufständischen gerichtet, 10.000e Polen, die der Beteiligung verdächtig waren, wurden nach Sibirien verbannt oder ins Exil getrieben, Grundbesitz verdächtiger Angehöriger des Adels wurde enteignet und vornehmlich an Russen übertragen, Polen wurden zugunsten von Russen aus örtlichen Leitungspositionen gedrängt. Das Polnische blieb allerdings Verwaltungssprache.

Diese Repressionspolitik dauerte in der geschilderten Schärfe bis zum Tode von Nikolaus I. 1855 und dem von Paskewitsch 1856 an. Danach setzte unter dem neuen Zaren und König von Polen, Alexander II., eine gewisse Entspannung ein, die 1857 in eine Amnestie und in eine (Wieder-)Zulassung polnischer Vereine und Organisationen mündete, darunter der Towarzystwo Rolnicze (Agrargesellschaft) mit vielen Niederlassungen innerhalb des Königreiches, in welcher nicht nur agrarische, sondern auch allgemeine Fragen diskutiert wurden, so dass sie sich den Ruf eines „Ersatzparlamentes“ erarbeitete. Insbesondere aus den weiter bestehenden unterschiedlichen Sichtweisen zwischen der ,roten“ und der ,weißen“ Fraktion des polnischen Adels, wie angesichts ihrer allmählichen Hinwendung zu polnischen Autonomiepositionen mit der russischen Herrschaft umgegangen und welche Ziele (Wiederinkraftsetzung des Organischen Statuts, der Verfassung von 1815 oder weitergehende Autonomie) verfolgt werden sollten, entwickelten sich aber Streitigkeiten und Demonstrationen, von denen eine im April 1861 durch Polizeitruppen blutig (mit 100 Toten) aufgelöst wurde. Gleichwohl erlangte in diesem Zeitraum der dem „roten“ Lager zuzurechnende Befürworter weitgehender Agrarreformen, der polnische Marquis Aleksander Wielopolski, einen herausragenden administrativen Status, indem er zunächst vom 1856 wiedereingesetzten Statthalter Russlands, Michail Gortschakow, zum Minister für Religions- und Bildungsangelegenheiten und 1862 vom Zaren zum Leiter der Zivilverwaltung, also zum faktischen Ministerpräsidenten des Königreiches ernannt worden war. Wielopolski geriet infolge seiner engen Kooperation mit der zaristischen Admi-

25 Siehe Fn. 24 (Zusatzkommentar a. E.). 
nistration aber zunehmend in die Kritik und unter Druck der polnischen Kreise, die für eine weitergehende Autonomie, letztlich auch für eine echte Souveränität kämpfen wollten. Die Unterstützung für dieses Postulat erfasste immer mehr Kreise der polnischen Bevölkerung, insbesondere des bislang eher abwartenden Teils des Adels, und auch in den von Preußen und Österreich besetzten Gebieten, was schließlich 1863 zur sog. Januarerhebung führte. Dabei wurden polnische Truppen von Romuald Traugutt befehligt, dem der zaristische Gegner den Beinamen „Diktator" anhängte, um ihn zu diskreditieren.

Dieser Januaraufstand endete in einem militärischen und politischen Fiasko. Zwar hatten sich anfänglich russische Truppen zurückgezogen, auch aus den früher zu Litauen gehörenden Territorien der jetzt dem russischen Kernland angegliederten „westlichen“ Gouvernements, aber die Aufständischen vermochten es nicht, ausreichende Breitenwirkung zu erzielen, weder in den Städten noch auf dem Lande. Und mit der Absetzung Wielopolskis und der Ernennung des baltisch-deutschen Feldmarschalls Friedrich ( фёдор) von Berg zum neuen Statthalter/Vizekönig gewann Russland ab Sommer 1863 wieder die Initiative zurück.

\section{C. „Weichselland““}

\section{Beginn der Depolonisierung}

Die Folge des gescheiterten Aufstandes war eine Wiederhinwendung der russischen Politik von einer eingeschränkten Akzeptanz des polnischen Sonderstatus, wie sie sich im Organischen Statut von 1832 manifestiert hatte (wenngleich ohne die früheren weitergehenden Autonomieregelungen der Schlussakte des Wiener Kongresses und der Verfassung von 1815), hin zu einer zumindest konstitutionellen Beseitigung jeglicher Selbstbestimmung. Außerdem versuchte die zaristische Administration, einen Keil zwischen den polnischen Adel als wesentliche Kraft des Aufstandes und den für den Aufstand nicht mobilisierbar gewesenen Stand der kleinen und mittleren Bauern zu treiben.

Nachdem der aufständische Adel mit drakonischen Straf- und Enteignungsmaßnahmen verfolgt worden war, die in der Hinrichtung der Symbolfigur der Rebellen, Traugutt, am 5. August 1864 gipfelten, und gleichzeitig die zaristische Administration eine Landreform initiiert hatte, um jegliche Solidarisierung der Bauern mit dem aufständischen Adel für die Zukunft zu verhindern, begannen die russischen Maßnahmen zur Aushöhlung bzw. endgültigen Abschaffung der konstitutionell immer noch - wenngleich nur rudimentär - vorhandenen polnischen Staatlichkeit. So wurde von Russland ein Үчреди́тельный Комитет [Gründungskomitee] eingesetzt, welches sich unter dem Vorsitz des russischen Statthalters/Vizekönigs mit der vorbeschriebenen Agrarfrage beschäftigen soll- 
te, das sich aber schnell zu einer organisatorischen Schaltzentrale der russischen Herrschaft entwickelte. Weil dieses Komitee unter anderem auch für weite Teile des Finanzwesens im Königreich zuständig war, hatte es erheblichen steuernden Einfluss auf die Polen betreffenden Entscheidungsprozesse.

\section{Vollzug der Depolonisierung}

Im Rahmen dieser „Verwaltungsreform“26 löste Russland zunehmend alle spezifisch polnischen Einrichtungen auf, die zur Administrierung des Königreiches berufen waren, ohne diese tiefgreifenden Maßnahmen an den Formalien und Regularien des immer noch formell geltenden Organischen Statuts zu orientieren, welches dadurch aber faktisch abgeschafft war. So erfolgte 1867 die Auflösung des Staats- und des Verwaltungsrates, nachdem man Strukturen etabliert hatte, die jenen der Kerngebiete des russischen Reiches glichen, indem die einzelnen Organisationsbereiche der Innen-/Polizei-, Finanz-, Justiz- und Bildungsverwaltung unmittelbar der russischen Zentralverwaltung unterstellt wurden. Schon 1866 war nämlich diese administrative Umstrukturierung weitgehend abgeschlossen, und nach der Aufteilung in zehn Gouvernements vermieden die zaristischen Instanzen fürderhin nicht nur die Bezeichnung „Königreich“, sondern sogar das Wort Polen; sie nannten das Territorium degradierend nur noch Привислинский край / Kraj Nadwiślański bzw. Kraj Przywiślański (Weichselland oder -gebiet). Dass in diesem Zusammenhang auch die Hauptstadt des Königreichs, Warschau, in ihrer Titulatur von Miasto stoleczne zu город herabgestuft wurde, war letztlich nur ein weiteres bezeichnendes Element der Abschaffung des polnischen Sonderstatus innerhalb Russlands. Auch wurde 1867 die bisherige Nationalhymne Mazurek Dabrowskiego durch die russische Боже, Царя храни! ersetzt. Dieser rigorosen Russifizierung der konstitutionellen Staatlichkeit Polens folgend entsprach es dann auch der russischen Politik, im Jahre 1874 nach dem Tode des zaristischen Statthalters von Berg, der bis dato noch den zehn von Russland ernannten Gouverneuren vorgestanden hatte, das Amt des Statthalters/Vizekönigs in dasjenige eines Generalgouverneurs umzuwandeln. Trotz dieser statusmäßigen Degradierung blieben die Generalgouverneure wie auch schon die Statthalter/Vizekönige Befehlshaber der etwa 240.000 Mann starken russischen Truppen in Polen, die schon während der Geltung der Verfassung des Königreichs die nur wenige Jahre eigenständige polnische Armee ersetzt hatten.

Mit dieser Depolonisierung der konstitutionellen Institutionen des Königreiches einher gingen auch entsprechende Maßnahmen auf regionaler und lokaler Ebene. So versuchte Russland, die seinerzeit verfassungsmäßig (§ 29), später aufgrund des Organischen Statuts (Art. 7 Satz 2) verankerten Rechte der Polen

26 A.N. Makarov, op. cit., S. $344 \mathrm{ff}$. 
auf Zugang zu fast allen öffentlichen Ämtern des Königreichs auszuhebeln und die entsprechende, besonders von Wielopolski betriebene Praxis umzukehren. Zwar waren die obersten Funktionen nur für orthodoxe/russische Amtsträger vorgesehen, die nunmehr eigentlich auch in subalterneren Ämtern nicht mehr gewollten Katholiken/Polen mussten aber immer wieder notgedrungen eingesetzt werden, weil die russische Beamtenschaft nicht zuletzt mangels Interesses insbesondere aus pekuniären Gründen die Vakanzen nicht ausgleichen konnte bzw. wollte. Ein weiteres wichtiges Element der Russifizierung war die Zurückdrängung des Polnischen als Amtssprache, entgegen den ausdrücklichen Regeln des Organischen Statuts (Art. 34); dabei traf diese Politik gerade auch die polnischen Schulen, in denen Russisch als 1. Unterrichtssprache sowie russische Geschichte und Literatur Priorität genossen, Polnisch war nur als Fremdsprache an Gymnasien zugelassen. Vergleichbares fand an den Universitäten statt. Der Besitz der katholischen Kirche wurde vielfach beschlagnahmt, einige Klöster wurden aufgelöst, die völlig neu geschaffenen Diözesen dem Katholischen Kollegium, einer Abteilung der russischen Staatsbehörde des Geistlichen Kollegiums unterstellt, auch insoweit ein klarer Verstoß gegen Art. 6 des Organischen Statuts. Straßenschilder wurden in kyrillischer Schrift angebracht, Städte russisch umbenannt.

Insgesamt gesehen, stand die Russifizierung als strategisches Ziel im Vordergrund, und um dieses zu erreichen, musste zunächst die Depolonisierung gelingen, deren Maßnahmen immer mehr anzogen. Die These, dass es Russland vordringlich „nur“ um letztere gegangen sei, machen manche Historiker an der Tatsache fest, dass die ersten Generalgouverneure gar keine Russen, sondern wie von Berg und sein Nachfolger von Kotzebue Deutsch-Balten waren. Immerhin waren diese auch und gerade Militärs in russischen Diensten, und ihre Nachfolger wie Josef Wladimirowitsch Gurko, Alexander Konstantinowitsch Imeretinski und Jakow Grigorjewitsch Schilinski wiederum ,,autochthone“ russische Militärs.

Diese Maßnahmen dauerten letztlich bis zum Wiedererstehen eines souveränen Staates Polen im Jahre 1918 in Folge des Ersten Weltkrieges an.

\section{Parallelen zur heutigen Situation?}

\section{Aussage von Staatspräsident Duda}

In einer Rede anlässlich des 100. Jahrestages dieses Ereignisses hatte der polnische Staatspräsident Andrzej Duda unlängst, nämlich am 13. März 2018 im niederschlesischen Kamienna Góra Parallelen zwischen der seit der Dritten polnischen Teilung 1795 substanziell erfolgten Beseitigung der polnischen Souveränität durch Preußen, Österreich und vornehmlich durch Russland und den heutigen 
Maßnahmen der EU, sich nationalstaatliche Kompetenzen nach „Brüssel“ zu holen oder diese durch europäische Rechtssetzung zu „überwölben“, angedeutet ${ }^{27}$ :

Denn sehr oft sagen die Leute: ,Warum brauchen wir Polen? Die Europäische Union ist das Wichtigste. ' Ihr wisst, dass es solche Stimmen gibt. Dann sollen sich alle diese Menschen an die 123 Jahre Besatzung/Aneignung erinnern, als Polen am Ende des 18. Jahrhunderts seine Unabhängigkeit verlor und von der Landkarte verschwand. Es gab dann auch diejenigen, die sagten: ,Vielleicht ist es besser so, es wird endlich keinen Streit mehr geben, keine Aufstände, keine Erhebungen, keine Kriege, keinen Zank, keine Konföderationen/Bündnisse, und schließlich wird es Frieden geben. Dann haben sie aber bald begriffen, dass es weiterhin Kriege und Streitigkeiten gibt, nun haben wir aber keinen Einfluss mehr und entscheiden nicht mehr über uns selbst, weil irgendwo in der Ferne, in entfernten Hauptstädten, über unsere Angelegenheiten entschieden wird...

Ausgehend von der Feststellung, dass im Jahre 1815 sowohl in der Wiener Schlussakte als auch in der Verfassung des Königreichs Polen zwar zunächst „nur" eine faktische Personalunion zwischen dem Staatsoberhaupt und Souverän des russischen Kaiserreichs und des Königreichs Polen, in Gestalt des russischen Zaren, oder zumindest eine unio inaequali jure ${ }^{28}$ konstituiert wurde, ergab der weitere historische Verlauf hingegen die schrittweise Degradierung eines zumindest teilsouveränen Polen hin zu dem Status eines russischen Provinzialgebildes. Diese Entstaatlichung Polens war allerdings umso leichter zu bewerkstelligen, als gerade im russischen Kaiserreich der Zar - gemessen an anderen europäischen Monarchen - mit einer besonders starken autokratischen Kompetenz des alleinigen Souveräns ausgestattet war, und zwar ohne Begrenzung durch einen konstitutionellen Rahmen.

Angesichts der aktuellen Situation, in der - neben anderen Staaten der Visegrád-Gruppe - insbesondere Polen in den kritisierenden Fokus von ernannten und selbsternannten Hütern Europas in EU-Kommission ${ }^{29}$, EU-Parlament ${ }^{30}$ und Europäischem Rat ${ }^{31}$, und auch in Regierungen ${ }^{32}$ und Parlamenten ${ }^{33}$ einiger EU-Mit-

27 http://wiadomosci.gazeta.pl/wiadomosci/7,114884,23139252,duda-w-kamiennej-gorze-porownal-ue-do-zaborcow-polityczna.html, zuletzt abgerufen am 14.12.2018; http://www.spiegel.de/ politik/ausland/polens-andrzej-duda-vergleicht-die-eu-mit-besatzungsmaechten-a-1198159.html, zuletzt abgerufen am 14.12.2018.

28 A.N. Makarov, op. cit., S. 336 f.

$29 \mathrm{http} / /$ europa.eu/rapid/press-release_IP-17-5367_de.htm, zuletzt abgerufen am 14.12.2018.

$30 \mathrm{http}: / /$ www.europarl.europa.eu/sides/getDoc.do?pubRef=-//EP//TEXT+TA+P8-TA-20170442+0+DOC+XML+V0//DE, zuletzt abgerufen am 14.12.2018.

31 http://www.consilium.europa.eu/de/meetings/gac/2018/02/27/, zuletzt abgerufen am 14.12.2018; https://ec.europa.eu/germany/news/20180227-verteidigung-des-rechtsstaats-polen_de, zuletzt abgerufen am 14.12.2018.

32 https://www.tagesspiegel.de/politik/eugh-urteil-zur-verteilung-von-fluechtlingen-asselborn-jedes-land-hat-sich-jetzt-daran-zu-halten/20289738.html, zuletzt abgerufen am 14.12.2018.

$33 \mathrm{http}: / / w w w . t h e n e w s . p l / 1 / 10 / A r t y k u l / 343021$,Germany-to-limit-contributions-to-EU-ifPoland-doesnt-accept-migrants-SPD-chief, zuletzt abgerufen am 14.12.2018. 
glieder geraten ist, hat nun Staatspräsident Duda es für erforderlich gehalten, in seiner an polnische Bürger gerichteten Rede Parallelen zu jener Zeit mangelnder Souveränität Polens zu ziehen, besser gesagt: anzudeuten.

\section{Fragestellung}

Worum geht es? Eine gewisse staatspolitische Zurückhaltung nicht so ausüben zu müssen wie es von dem Staatsoberhaupt eines demokratischen Staates und Mitglieds der EU erwartet wird, und die in seiner Rede in Kamienna Góra angedeuteten Feststellungen zu vertiefen, könnten dazu führen, in Bezug auf die Geschehnisse ab 1815 ff. und die heutigen ab 2015 ff. folgende Fragen zu stellen:

Begegnet Polen, dessen mutige Bürger als erste im Gebiet des damaligen Warschauer Paktes das Fanal für den Zusammenbruch des kommunistischen Systems und damit für ein geeintes Europa gesetzt haben, heutzutage angesichts der Kritik einiger westeuropäischer Partner am polnischen Umgang mit der Migrationskrise sowie an der polnischen Justizreform einem vergleichbaren Undank wie nach seiner entscheidenden Hilfe für das seinerzeit seit Jahrhunderten latent, Ende des 17. Jahrhunderts erneut akut vom aggressiven Osmanentum bedrohte Westeuropa ${ }^{34}$ ?

Äußert sich diese Undankbarkeit angesichts des innereuropäischen Drucks durch die zuständigen EU-Institutionen und die sie insoweit dominierenden Mitgliedsstaaten, die überwiegend muslimischen Migranten auch nach Polen zu verteilen, ebenso darin, die bitteren historischen Erfahrungen Polens und anderer Länder insbesondere der Visegrád-Gruppe mit den seinerzeitigen ${ }^{35}$ und heutigen ${ }^{36}$ Domi- $^{-}$ nanzbestrebungen aus vornehmlich muslimischen Kulturkreisen zu ignorieren?

Wie ist in diesem Zusammenhang die Aussage des derzeitigen Präsidenten der EU-Kommission, Jean-Claude Juncker, zu bewerten, die er seinerzeit als luxemburgischer Ministerpräsident und Finanzminister getroffen hatte?: ,Wir beschließen etwas, stellen das dann in den Raum und warten einige Zeit ab, was passiert. Wenn es dann kein großes Geschrei gibt und keine Aufstände, weil die meisten gar nicht begreifen, was da beschlossen wurde, dann machen wir weiter — Schritt für Schritt, bis es kein Zurück mehr gibt.، ${ }^{\text {(37 }}$

${ }^{34} \mathrm{http} / / /$ ieg-ego.eu/de/threads/modelle-und-stereotypen/tuerkengefahr-exotismus-orientalismus/markus-koller-die-osmanische-geschichte-suedosteuropas, zuletzt abgerufen am 14.12.2018.

35 s.o. Fn. 1) und 2).

36 https://www.welt.de/geschichte/article159307886/Dieses-Imperium-ist-Erdogans-grosses-Vorbild.html, zuletzt abgerufen am 14.12.2018; http://www.spiegel.de/politik/ausland/tuerkei-recep-tayyip-erdogan-traeumt-vom-osmanischen-reich-a-1118342.html, zuletzt abgerufen am 14.12.2018; https://www.deutschlandfunk.de/historisches-grenzabkommen-ankara-stellt-tuerkisch.795.de.html?dram:article_id=370648, zuletzt abgerufen am 14.12.2018; https://www.faz. net/aktuell/feuilleton/debatten/streit-um-ditib-ein-undurchsichtiges-spiel-14394916.html, zuletzt abgerufen am 14.12.2018.

37 Der SPIEGEL, Die Brüsseler Republik, 27.12.1999, http://www.spiegel.de/spiegel/ print/d-15317086.html, zuletzt abgerufen am 14.12.2018. 


\section{Maßnahmen der EU gegen Polen}

Gegenstand der nunmehr gegen Polen gerichteten zweiten „Maßnahmen der Kommission zum Schutz der Rechtsstaatlichkeit" gem. Art. 7 EUV ${ }^{38}$ wegen von den EU-Gremien behaupteten Verstößen gegen Tatbestände des Wertekanons des Art. 2 EUV sind die aktuellen polnischen Gesetze zur Justizreform. „Zweite“ Maßnahmen deshalb, weil es bislang im Jahre 2000 erst ein mit ähnlicher Zielsetzung begonnenes, relativ weit gediehenes, aber zurückgenommenes Verfahren gegen Österreich (,Sanktionen der XIV“) gab; dieses war schon damals, zumindest aber nach den Aktualisierungen des und den Ergänzungen zum EUV erst recht als unzulässig zu qualifizieren ${ }^{39}$. Im Hinblick auf Polen hatte die EU-Kommission Ende 2017 in der sog. Präventionsphase gem. Art. 7 Abs. 1 EUV im Wesentlichen vorgeschlagen, die neuen polnischen gesetzlichen Bestimmungen über die verkürzte Amtsdauer von Richtern mit der Befugnis des polnischen Staatspräsidenten, diese im Einzelfall wieder zu verlängern, über die richterlichen Besetzungen des Justizrates und über die Besetzung des polnischen Verfassungsgerichts zu beanstanden ${ }^{40}$. Inwieweit diese Maßnahmen weiter betrieben werden bzw. letztlich zu einem Beschluss nach Art. 7 Abs. 3 EUV (Ausschluss/Aussetzung noch zu bestimmender Rechte, insbesondere des Stimmrechtes Polens im Europäischen Rat und/oder im Rat) führen, bleibt abzuwarten. Jedenfalls müsste einem derartigen Beschluss eine einstimmige Feststellung des Europäischen Rates im Sinne des Art. 7 Abs. 2 EUV voraufgehen, wobei nach Art. 354 Abs. 1 Satz 1 AEUV ${ }^{41}$ Polen nicht stimmberechtigt wäre. Ob eine derartige Einstimmigkeit angesichts der politischen Gegebenheiten insbesondere in den anderen Visegrád-Staaten, neuerdings auch in Italien, erzielt werden würde, dürfte — gerade auch nach Verlautbarungen aus Ungarn - sehr zweifelhaft sein.

Bei einem anderen Gegenstand der Kritik an Polen, die von Gremien der EU und einigen der Regierungen ihrer Mitgliedsstaaten, darunter besonders vehement - auch innenpolitisch — von derjenigen des Heimatlandes des Autors ${ }^{42}$, hervorgebracht wird, ist die Haltung Polens zur „Migrationskrise“. Hier wehrten sich Ungarn und die Slowakei schon gegen den Beschluss des Rates der Innen-

38 https://eur-lex.europa.eu/legal-content/EN/ALL/?uri=OJ\%3AC\%3A2012\%3A326\%3AT OC, zuletzt abgerufen am 14.12.2018.

$39 \mathrm{https} / / /$ images.derstandard.at/upload/images/bericht.pdf, zuletzt abgerufen am 14.12.2018.

$40 \mathrm{http}$ ://ec.europa.eu/newsroom/just/document.cfm?action=display\&doc_id=49108, zuletzt abgerufen am 14.12.2018.

41 https:/eur-lex.europa.eu/legal-content/EN/ALL/?uri=OJ\%3AC\%3A2012\%3A326\%3AT OC, zuletzt abgerufen am 14.12.2018.

42 https://www.wiwo.de/politik/deutschland/regierungserklaerung-merkel-legt-sich-mitungarn-und-polen-an/20991808.html, zuletzt abgerufen am 14.12.2018; https://www.zeit.de/ news/2018-02/22/merkel-willeu-gelder-an-fluechtlingsaufnahme-knuepfen-180221-99-182639, zuletzt abgerufen am 22.06.2018; http:/www.dw.com/de/gesine-schwan-die-spd-kann-merkelparoli-bieten/a-43010982, zuletzt abgerufen am 17.12.2018. 
minister aus dem Jahr 2015, der auf Vorschlag der EU-Kommission und mit Zustimmung der Mehrheit des EU-Parlamentes getroffen worden war, dagegen, im Rahmen der Umsiedlung von 120.000 und nochmals 40.000 irregulär nach Italien und Griechenland eingereisten Migranten ${ }^{43}$ in die anderen EU-Mitgliedsstaaten, „ihre“ Kontingente von mehreren 1000 Menschen aufnehmen zu müssen. Auch Polen hatte die Entscheidung des Rates nicht umgesetzt. Die Kommission bzw. der Rat hatten geltend gemacht, dass es sich bei dieser Verteilaktion um eine Maßnahme zugunsten der durch die Mittelmeer- und Balkan-Wanderungsbewegungen besonders belasteten Staaten Italien und Griechenland handele, auf die durch Ausübung der Notlagenkompetenz des Art. 78 Abs. 3 AEUV reagiert werden müsse. Klagen von Ungarn und der Slowakei gegen den Beschluss des Rates hat der EuGH mit Urteilen vom 6.09.2017 ${ }^{44}$ abgewiesen, denn der Tatbestand des Art. 78 Abs. 3 AEUV sei erfüllt. Die Regelung sei eine nur „vorläufige“, obwohl für 200.000 Fälle endgültige Zuweisungen stattfinden sollen; auch müsse ein „plötzlicher Zustrom aus Drittländern“ nicht unvorhersehbar gewesen sein. Polen hatte nicht geklagt, sondern wie die Tschechei den Beschluss einfach nicht umgesetzt, auch weil es bereits Hunderttausende Ukrainer als Folge der Krise in der Ostukraine beherbergt und es der Aufnahme von Muslimen, die den Hauptteil der Migranten ausmachen, wohl auch ablehnend gegenübersteht. Am 14.06.2017 hat die EU-Kommission daraufhin ein Vertragsverletzungsverfahren gegen Polen, Ungarn und Tschechien - gleichzeitig mit einem entsprechenden Verfahren gegen Deutschland und Österreich wegen Versäumnissen von Berichtspflichten aus der EU-Abfallrichtlinie - eingeleitet ${ }^{45}$. Mangels aus Sicht der EU-Kommission zufriedenstellender Antworten der drei betroffenen Länder in der Migrationsfrage, die eine Anrufung des EuGH hätten vermeiden können, erhob diese am 7.12.2017 Klage vor dem EuGH ${ }^{46}$. Auch der Ausgang dieses Verfahrens bleibt abzuwarten.

Derzeit, im 100. Jahr der Wiedererlangung seiner vollen staatlichen Souveränität, sieht sich also Polen besonderen europarechtlich basierten „Disziplinierungsbestrebungen“ der EU ausgesetzt, die den polnischen Staatspräsidenten veranlasst haben, zugegebenermaßen etwas verklausuliert, Parallelen zu der Zeit der vorhergehenden Zarenherrschaft über polnische(s) (Kern-)Staatsgebiet, -volk und -gewalt, die bis zum Ende des 1. Weltkrieges andauerte, anzusprechen.

43 https://eur-lex.europa.eu/legal-content/EN/TXT/?uri=OJ\%3AJOL_2015_239_R_0011 und https:/eur-lex.europa.eu/legal-content/EN/TXT/?uri=CELEX\%3A32015D1601, jeweils zuletzt abgerufen am 14.12.2018.

44 Urteile C-643/15 und C-647/15, http://curia.europa.eu/juris/liste.jsf?num=C-643/15\&language $=$ de, zuletzt abgerufen am 14.12.2018.

45 https://ec.europa.eu/germany/news/vertragsverletzungsverfahren-gegen-tschechien-ungarn-und-polen-wegen-fl\%C3\%BCchtlingspolitik-gegen_de, http://europa.eu/rapid/press-release_ IP-17-1607_de.htm, jeweils zuletzt abgerufen am 14.12.2018.

46 http://europa.eu/rapid/press-release_IP-17-5002_de.htm, zuletzt abgerufen am 14.12.2018. 


\section{Bewertung}

\subsection{Formelle Parallelität}

Nur: Ist diese Sicht gerechtfertigt? Sicherlich nicht hinsichtlich der Frage, wie sich die polnische Entstaatlichung bzw. die Übertragung polnischer staatlicher Kompetenzen seit 1795, insbesondere seit 1815, im Vergleich zu den Geschehnissen seit dem Beitritt Polens zur EU gestaltet hatten. Denn die Verhältnisse beider Epochen unterscheiden sich doch wesentlich: Während seit 1795 fremde Akteure, insbesondere die kaiserlichen Souveräne in St. Petersburg seit 1815, hinsichtlich weiterer Gebiete, die vor 1792 ebenfalls zu Polen gehörten, auch die Monarchen in Preußen und Österreich, letztendlich darüber entschieden haben, ob und wie sich Polen seiner Souveränität zu begeben hat, erfolgte mit dem Beitritt zur EU die Abgabe staatlicher Kompetenzen nach „Brüssel“ durch den jetzigen Souverän, das polnische Volk. Dieses hatte den Beitritt im Referendum vom 7. und 8. Juni $2003^{47}$ demokratisch beschlossen und damit die Inhalte des EUV, des AEUV und des weiteren Europarechtes vollinhaltlich akzeptiert, zu denen auch gehört, Teile der Staatsgewalt auf das supranationale Gebilde EU zu übertragen. Dass jedenfalls auch ein entsprechender actus contrarius gem. Art. 50 EUV kraft freien Willen des Volkes bzw. seiner demokratisch legitimierten Institutionen möglich wäre, zeigt das Beispiel des Vereinigten Königreichs von Großbritannien und Nordirland.

\subsection{Inhaltliche Parallelität — Grundlagen}

Die Frage darf aber gestellt werden, wie weit die Souveränitätsrechte auf die supranationalen Institutionen der EU verlagert werden dürfen bzw. sollen, insbesondere solche identitären Rechte, die nach dem jeweiligen nationalen Verständnis zum nicht entäußerungsfähigen Kernbereich der nationalen staatlichen Kompetenz gehören bzw. gehören sollten. Als allgemeiner Ausdruck dieses Gedankens gilt „eigentlich“ in der EU das sog. Subsidiaritätsprinzip, verankert in Art. 5 Abs. 3 EUV, in dem Protokoll (Nr. 2) über die Anwendung der Grundsätze der Subsidiarität und der Verhältnismäßigkeit (Subsidiaritätsprotokoll) ${ }^{48}$, darüber hinaus in Art. 12 Buchst. b) EUV und wohl auch in Art. 5 Abs. 2 Satz 2 EUV, mit den Möglichkeiten der Subsidiaritätsrüge und -klage ${ }^{49}$.

In diesem Zusammenhang hat natürlich die Aussage des jetzigen EU-Kommissionspräsidenten Bedeutung (s. o. D. 2.), die jener noch als Amtswalter eines Mitgliedstaates getroffen hatte, der sich in diesem Zeitraum mit steuerlichen Rege-

47 https://pl.wikipedia.org/wiki/Referendum_w_Polsce_w_2003_roku, zuletzt abgerufen am 14.12.2018.

48 https://eur-lex.europa.eu/legal-content/DE/TXT/?uri=CELEX:12008E/PRO/02, zuletzt abgerufen am 14.12.2018.

$49 \mathrm{http} / / / \mathrm{www}$.infopoint-europa.de/assets/vonZezschwitz-Kompetenzausubungsregeln-EU. pdf, S. 5, zuletzt abgerufen am 14.12.2018. 
lungen hervorgetan hatte, die als besonders unsolidarisch zu anderen EU-Mitgliedsstaaten und unlauter gegenüber dem Gros „normaler“ europäischer Steuer zahlender natürlicher und juristischer Personen qualifiziert werden müssen („Steueroase“ für überseeische Konzerne $)^{50}$. Sein Zitat muss als Beschreibung einer „Salamitaktik“ verstanden werden, die darauf gerichtet ist, sukzessive unter Außerachtlassung des Subsidiaritätsprinzips immer weitere Kompetenzen der Mitgliedsstaaten nach Brüssel zu hieven, ohne dass die Parlamente (EU und nationale), geschweige denn die europäischen Bevölkerungen diese Aushöhlung merken oder merken sollen. Ein klandestines Verhalten, das den Urheber der Äußerung eigentlich für jedes politische Amt zumindest auf europäischer Ebene hätte unwählbar machen müssen.

Zur Beurteilung des polnischen Verständnisses über derartige neuzeitliche Taktiken der Preisgabe nationaler Souveränität und deren europarechtlicher Zulässigkeit ist daher die Einbeziehung der historisch-politischen Erfahrungen Polens unabdingbar. Wie die Positionierung Ungarns und der Slowakei zur Frage des Zustroms von vornehmlich aus dem islamischen Kulturkreis stammenden illegalen Migranten wohl nicht zuletzt auf dem historischen Bewusstsein dieser Völker über die in Ungarn von 1526 bis etwa 1720 und in der Slowakei von 1542 bis 1685 herrschende osmanische Besetzung mit verheerenden Folgen (Zwangskonversionen, Sklaverei, erheblicher Rückgang der Bevölkerung) ${ }^{51}$ beruht und von den EU-Gremien entsprechend hätte berücksichtigt werden müssen, sollte sich das „ferne“ Brüssel bei seinen Maßnahmen gegen den neuen institutionellen, insbesondere justiziellen Staatsaufbau Polens des Umgangs nichtpolnischer Autoritäten mit der polnischen Souveränität und Identität in jener Epoche der Nichtexistenz eines souveränen Polen dringend bewusst werden. Auch die polnischen Leistungen bei der Bewahrung Westeuropas 1683 vor einem osmanischen Joch dürfen nicht ausgeblendet werden. Dass gerade das EU-Mitglied Deutschland in Verantwortung für seine in den überfallenen Mitgliedsländern der EU, insbesondere in Polen, während der deutschen Besetzung 1939-1944/45 begangenen Verbrechen gegen den Frieden und gegen die Menschlichkeit ${ }^{52}$ hier besondere Zurückhaltung üben müsste, sollte eigentlich selbstverständlich sein, ist es aber leider nicht.

Die EU-Gremien und viele der EU-Mitgliedstaaten versuchen derzeit, auch begünstigt durch die stark ,,integrationsfreundliche“ Rechtsprechung des EuGH ${ }^{53}$,

50 http://www.sueddeutsche.de/wirtschaft/luxemburg-leaks-aerger-im-steuer-maerchenland-1.2206040, https://de.wikipedia.org/wiki/Luxemburg-Leaks\#Unter_Premier_Juncker_ (1995\%E2\%80\%932013), jeweils zuletzt abgerufen am 14.12.2018.

$51 \mathrm{http} / / /$ ieg-ego.eu/de/threads/crossroads/religionsraeume-und-konfessionsraeume/islam-ineuropa/patrick-franke-der-islam-staat-und-religion-im-europa-der-neuzeit, https://de.wikipedia. org/wiki/T\%C3\%BCrkenkriege, jeweils zuletzt abgerufen am 14.12.2018.

52 http://www.zeno.org/Geschichte/M/Der+N\%C3\%BCrnberger+Proze $\% \mathrm{C} 3 \% 9 \mathrm{~F}$, zuletzt abgerufen am 14.12.2018.

53 Ch. Calliess, Grundsatz der Subsidiarität: Nur ein leeres Versprechen?, https://www.unitrier.de/fileadmin/fb5/inst/IRP/BG_Einzeldokumente_ab_2010/BG_54/Vorabversion_BG54_05_ Calliess.pdf, zu D., vorletzte Seite, zuletzt abgerufen am 14.12.2018. 
die dem Subsidiaritätsprinzip nicht immer die gebotene Beachtung schenkt ${ }^{54}$, unter dem Deckmantel der angeblich erforderlichen Sicherung, tieferen Herstellung und Weiterentwicklung einer „Einheit“ Europas ${ }^{55}$ gegen mehr oder weniger autoritäre, ökonomisch und/oder militärisch prosperierende sowie „populistische“ Partner-, gegnerische oder konkurrierende Länder und Blöcke die historisch gewachsenen, verfassungsmäßigen, kulturellen und soziologischen Unterschiede unter einem bürokratisch-technokratischen Wust von europarechtlichen Petitessen einerseits und grundsätzlichen, nur gemeinschaftlich zu regelnden Tatbeständen andererseits einzuebnen, dabei aber grundlegende Werte des EUV tunlichst zu übersehen oder hintanzustellen. In diesem Kontext ist es natürlich nicht verwunderlich, dass das de Gaulle'sche „Europa der Vaterländer“ eilfertig in die Nähe von Nationalismus und Rechtsextremismus gerückt wird ${ }^{56}$, wobei die Gegner dieses Begriffs und seines Inhaltes aber außer Acht lassen, dass gerade de Gaulle entscheidende Schritte für eine Aussöhnung der ehemaligen „Erzfeinde“ Deutschland und Frankreich unternommen hatte ${ }^{57}$.

\subsection{Inhaltliche Parallelität - Betrachtung anhand des EUV}

Auf der europäischen Ebene besteht eine Hierarchie zwischen primärem Unionsrecht, wozu im Wesentlichen EUV und AEUV zählen, und sekundärem, wozu die unmittelbar verbindlichen Verordnungen (allgemein) und Beschlüsse (an die jeweiligen Adressaten gerichtet) sowie noch national umzusetzende Richtlinien gehören, Art. 288 AEUV, welche sich an dem Primärrecht zu orientieren haben ${ }^{58}$. Dabei obliegt ausschließlich dem EuGH die Verwerfungskompetenz für sekundäres Unionsrecht, welches mit dem Primärrecht, eben auch dem Subsidiaritätsprinzip, nicht vereinbar ist ${ }^{59}$.

Angesichts dessen:

Wie verhält es sich mit dem Gebot in Art. 3 Abs. 2 EUV, den EU-Bürgern „einen Raum der Freiheit, der Sicherheit und des Rechts ohne Binnengrenzen“ zu bieten, ,in dem - in Verbindung mit geeigneten Maßnahmen in Bezug auf die

54 Ch. Callies, op. cit., zu C. III. 1.

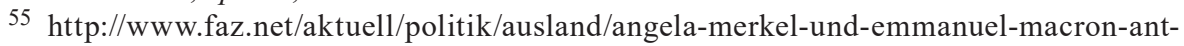
worten-auf-neugruendung-europas-15620502.html, zuletzt abgerufen am 17.12.2018; https://www. handelsblatt.com/archiv/davos-eroeffnung-die-rede-von-kanzlerin-merkel-im-wortlaut/2760382. html, zuletzt abgerufen am 17.12.2018.

56 http://www.bpb.de/nachschlagen/lexika/das-europalexikon/176853/europa-der-vaterlaender, zuletzt abgerufen am 17.12.2018, http://www.ina.fr/video/I00012372, zuletzt abgerufen am 17.12.2018.

57 https://www.youtube.com/watch?v=C-ThU4NG9EU, zuletzt abgerufen am 17.12.2018; http://www.degaulle.lpb-bw.de/6464.html, zuletzt abgerufen am 17.12.2018.

58 Siehe Übersicht, [in:] P. Stelkens, H.J. Bonk, M. Sachs, Verwaltungsverfahrensgesetz, 9. Aufl. 2018, 2. Teil, II.1.b) Rdnr. 38 ff., https://beck-online.beck.de/?vpath=bibdata/komm/StelBoSaKoVwVfG_9/cont/StelBoSaKoVwVfG.EuR.glII.gll.glb.htm, zuletzt abgerufen am 17.12.2018.

59 P. Stelkens, H.J. Bonk, M. Sachs, op. cit., Rdnr. 38 m.w.N. 
Kontrollen an den Außengrenzen, das Asyl, die Einwanderung sowie die Verhütung und Bekämpfung der Kriminalität — der freie Personenverkehr gewährleistet ist"? Vergleichbares gilt im Hinblick auf Art. 67 Abs. 1 AEUV, nach dem die Union ,einen Raum der Freiheit, der Sicherheit und des Rechts, in dem die Grundrechte und die verschiedenen Rechtsordnungen und -traditionen der Mitgliedstaaten geachtet werden", bildet bzw. zu bilden hat. Gleichzeitig ist ein verworrenes, insbesondere in Deutschland unterschiedlich ausgelegtes ${ }^{60}$ Geflecht aus internationalen, sekundär europarechtlichen sowie national kodifizierten und mündlich angewiesenen Zuwanderungs-, Asyl-, Seerechts- und Verfahrensbestimmungen ${ }^{61}$ entstanden, darunter der UN-Migrationsplan ${ }^{62}$, die EU-Migrationsagenda ${ }^{63}$ und ihre Fortschreibungen. Das ist verbunden mit den entsprechenden (Nicht- oder Fehl-)Umsetzungen in den hauptsächlich betroffenen Mitgliedsstaaten — gerade auch in Deutschland (Unzureichende Registrierungen und Identitätsfeststellungen, Zuwachs von Gewaltkriminalität, BAMF-Krise, Regierungsstreit über Grenzzurückweisungen von Migranten, die bereits mit einer Einreisesperre belegt oder als Asylsuchende in anderen Mitgliedsländern registriert wurden, usw.). Diese teilweise als "Staatsversagen“ charakterisierte Situation hat zu einem beispiellosen administrativen und sicherheitsgefährdenden Desaster sowie zu exorbitanten Kosten geführt. Davon sind gegenwärtig nur diejenigen Mitgliedsstaaten bislang verschont geblieben, die sich einer faktisch grenzen- und voraussetzungslosen Zuwanderung soweit wie möglich erwehren. Und nicht zuletzt droht diese zunehmende Problemoder sogar Krisenlage bei einigen Mitgliedsstaaten die EU zu spalten, was gerade beim „kleinen EU-Gipfel“ von 16 (willigen?) Mitgliedsländern am 24. Juni 2018 offenkundig geworden ist; das Ergebnis der Sitzung des Europäischen Rates am 28./29. Juni 2018 stand bei Schluss dieses Artikels noch nicht fest.

Wie kann bzw. soll die EU das in Art. 3 Abs. 3 Unterabs. 3 EUV verankerte Ziel erreichen, den Reichtum der kulturellen und sprachlichen Vielfalt Europas zu wahren und für den Schutz und die Entwicklung seines kulturellen Erbes zu sorgen, während immer weitere Migration geduldet, verteilt oder sogar gefördert wird, hauptsächlich aus einem bestimmten ferneren Kulturkreis, dessen poli-

${ }^{60}$ http://www.faz.net/aktuell/politik/fluechtlingskrise/fluechtlingskrise-in-deutschlandrechtsstaatlich-machbar-14060376.html?printPagedArticle=true\#pageIndex_0, zuletzt abgerufen am 17.12.2018. https://verfassungsblog.de/der-rechtsbruch-mythos-und-wie-man-ihn-widerlegt/, https://verfassungsblog.de/asyl-und-migration-recht-und-wirklichkeit/, jeweils zuletzt abgerufen am 17.12.2018.

61 Beispielhaft: http://ec.europa.eu/transparency/regdoc/rep/1/2015/DE/1-2015-240-DE-F1-1. PDF, https://eur-lex.europa.eu/legal-content/DE/TXT/HTML/?uri=CELEX:32016R1624\&from= DE, zuletzt abgerufen am 17.12.2018.

62 https://refugeesmigrants.un.org/sites/default/files/180711_final_draft_0.pdf, zuletzt abgerufen am 17.12.2018.

$63 \mathrm{https} / /$ ec.europa.eu/home-affairs/sites/homeaffairs/files/what-we-do/policies/europeanagenda-migration/background-information/docs/communication_on_the_european_agenda_on_ migration_en.pdf, zuletzt abgerufen am 17.12.2018. 
tisch-religiöse Avantgarden und Eliten schon in der Vergangenheit bewiesen und versucht haben und auch heutzutage oftmals unmittelbar oder über von ihnen platzierte NGOs versuchen, das kulturelle, also christlich-jüdisch-humanistische Erbe und den diesbezüglichen Anspruch Europas zugunsten eines im Mittelalter verharrenden totalitären Menschen-, Welt- und Gottesbildes zu relativieren oder gar zu unterdrücken und entsprechend umzugestalten, was leider in bestimmten Quartieren (,,no-go-areas“) west- und nord-europäischer Städte auch bereits eingetreten ist? Das vorgenannte Ziel spricht von Wahrung der (vorhandenen) europäischen Kulturvielfalt und Weiterentwicklung des (vorhandenen) Erbes, nicht aber von Implementierung neuer und fernerer Kulturen.

Diese Fragestellung ist angesichts besagter Kultur gerade auch für den elementaren Wert der Gleichberechtigung von Frauen und Männern, aufgeführt im Kanon des Art. 2 EUV und in Art. 3 Abs. 3 Unterabs. 2 EUV, ebenfalls besonders relevant.

Wird hinsichtlich des von der EU gegen Polen eingeleiteten Verfahrens wegen behaupteter Verletzung des Rechtsstaatsprinzips des Art. 2 EUV durch seine neuen Justizgesetze mit zweierlei Maß gemessen? Erfolgt doch die Ernennung der Richter bei den europäischen Gerichten selbst, also derzeit dem EuGH und dem europäischen Dienstgericht, gem. Art. 19 Abs. 2 Unterabs. 3 Satz 2 EUV ausschließlich durch die Regierungen der Mitgliedsstaaten, wenn auch im gegenseitigen Einvernehmen und gem. Art. 255 AEUV nach Bewerberprüfung durch einen siebenköpfigen Ausschuss, von dem allerdings nur ein Mitglied durch das EU-Parlament gewählt wird.

Alle EU-Mitgliedsstaaten sind gehalten, den EURO einzuführen, Art. 3 Abs. 4 EUV. Zu seinem Glück hat sich Polen diesem Postulat bislang versagt ${ }^{64}$, so dass es den teilweise eingetretenen Risiken für die nationalen Budgets aller und die ökonomische Entwicklung einiger Länder der Eurozone, die durch die jüngsten Vorschläge z.B. aus Frankreich („Europäischer Finanzminister“, „Eurozonen-Budget" $^{65}$ und der EU selbst („Plastiksteuer“ als eigenes EU-Steuerfindungsrecht) ${ }^{66}$ noch steigen könnten, bislang entgangen ist. Auch dieses wohl eher politisch, weniger ökonomisch begründete Konstrukt bedeutet eine Übertragung nationaler Souveränität, zu deren Kernbereich eben das Budgetrecht und die eigene Währung gehören, auf supranationale Institutionen. Letztere, z.B. die EZB, übt durch

${ }^{64}$ L. Knopp, M. Zgolak, Polen in die Eurozone - Vor dem Hintergrund fehlgeleiteter deutscher „Rettungspolitik“, WiRO 2013, $360 \mathrm{ff}$, https://beck-online.beck.de/Dokument?vpath=bibdata\%2Fzeits\%2Fwiro\%2F2013\%2Fcont\%2Fwiro.2013.360.1.htm\&anchor=Y-300-Z-WIRO-B-2013 -S-360, zuletzt abgerufen am 17.12.2018.

65 https://www.zeit.de/politik/ausland/2017-06/europaeische-union-reform-deutschlandfrankreich-emmanuel-macron/komplettansicht, zuletzt abgerufen am 17.12.2018, http://www.faz. net/agenturmeldungen/dpa/merkel-einigung-mit-macron-auf-eu-reformschritte-15648379.html, zuletzt abgerufen am 22.06.2018.

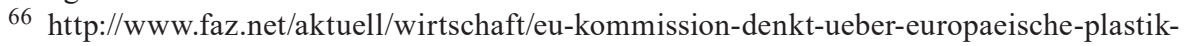
steuer-nach-15384372.html, zuletzt abgerufen am 17.12.2018. 
den dauernden Ankauf von Staatspapieren nicht premiumgerankter Länder der Eurozone im Werte von monatlich zig Milliarden Euro diese Kompetenzen auch extensiv aus ${ }^{67}$.

\section{E. Fazit}

Man kann es Präsident Duda nicht verdenken, dass er angesichts aktueller Maßnahmen der EU-Institutionen zu Recht Parallelen bei Angelegenheiten empfunden und angesprochen hat, die die nationale Souveränität und Identität Polens berührten und berühren, aber von „,fernen“ Autoritäten getroffen werden. Allerdings ist zunächst im rechtshistorischen Kontext zu bewerten, wie und wodurch diese fernen Autoritäten ihre Entscheidungsgewalt erhalten haben. Während der polnische Souverän der Jahre bis 1815 noch der polnische König (bzw. in der napoleonischen Ära der Herzog von Warschau) war, nahm dessen Amt ab 1815 kraft internationalen Verträgen, denen keine polnische Institution zugestimmt hatte, in Personalunion der russische Zar wahr; demgegenüber hat der heutige Souverän, das polnische Volk, vor 15 Jahren in freiem Willen seinen Beitritt zur EU und damit die Hingabe eines Teils seiner Souveränität an diese beschlossen. Inwieweit die primärrechtlichen Vertragswerke der EU alle bisherigen sekundärrechtlichen Entscheidungen ihrer Gremien, von denen sich Polen beeinträchtigt sieht, begründen oder rechtfertigen können, muss letztlich auch wieder eine europäische Institution, der EuGH, entscheiden, da diesem das Monopol zukommt, sekundäres Unionsrecht am primären zu messen. Durch Art. 50 EUV bestünde die theoretische und bisher nur von einem EU-Mitglied („Brexit“) wahrgenommene Möglichkeit, die nationale Souveränität wieder vollinhaltlich herzustellen.

\section{Literaturverzeichnis}

Calliess Ch., Grundsatz der Subsidiarität: Nur ein leeres Versprechen?, [in:] Stiftung Gesellschaft für Rechtspolitik (Hrsg.), Bitburger Gespräche Jahrbuch 2011 Bd. 1, München 2012.

Knopp L., Zgolak M., Polen in die Eurozone? - Vor dem Hintergrund fehlgeleiteter deutscher ,,Rettungspolitik“, WiRO 2013.

Makarov A.N., Die russisch-polnischen Rechtsbeziehungen seit 1815 unter spezieller Berücksichtigung der Staatsangehörigkeitsfragen, ZaöRV 1929.

Malte R., Imperiale Herrschaft im Weichselland: das Königreich Polen im Russischen Imperium (1864-1915), Berlin-München-Boston 2015, insbesondere auf Teil II, https://books.google.de/ books?id=hS11BgAAQBAJ\&pg=PA436\&lpg=PA436\&dq=weichselland\&source=bl\&ots=O

67 https://de.statista.com/statistik/daten/studie/241285/umfrage/bestand-der-von-der-ezb-im-rahmen-des-smp-aufgekauften-staatsanleihen/, zuletzt abgerufen am 17.12.2018. 
Y9-RsIfLA\&sig=a2XOsNSWlcV7UoUsYGnmRrZXPhY\&hl=de\&sa=X\&ved=0ahUKEwjN o5KVwrnbAhUEZ1AKHTdTDZcQ6AEIbzAM\#v=onepage $\& \mathrm{q}=$ weichselland\&f=false, $\mathrm{zu}-$ letzt abgerufen am 14.12.2018.

Stelkens P., Bonk H.J., Sachs M., Verwaltungsverfahrensgesetz: VwVfG, 9. Aufl. 2018.

\title{
The gradual abandonment of the nation-state: From "Congress Poland" to the "Vistula Gubernia" - are there any parallels with regard to EU legislation and its implementation? Reflections on state sovereignty and identity in relation to supranational law using the example of Poland
}

\begin{abstract}
Summary
In the light of Poland's historical experience of more than 100 years under foreign sovereignty, especially that of the Russian from 1815 to 1918, Poland's current reservations about the danger of an excessive transfer of national sovereign rights to European Union institutions. In the view of the undersigned, partial aspects of this transfer affect certain principles of European primary law.
\end{abstract}

Keywords: sovereignty, national state, culture, European Union, foreign rule. 